\title{
Kernos
}

Revue internationale et pluridisciplinaire de religion grecque antique

13 | 2000

Varia

\section{Paola CECCARELLI, La pirrica nell'antichità greco romana. Studi sulla danza armata}

\section{Alexandra Goulaki-Voutira}

\section{OpenEdition \\ Journals}

\section{Édition électronique}

URL : http://journals.openedition.org/kernos/1319

DOI : 10.4000/kernos.1319

ISSN : 2034-7871

\section{Éditeur}

Centre international d'étude de la religion grecque antique

Édition imprimée

Date de publication : 1 janvier 2000

ISSN : 0776-3824

\section{Référence électronique}

Alexandra Goulaki-Voutira, « Paola cECCARELLI, La pirrica nell'antichità greco romana. Studi sulla danza armata », Kernos [En ligne], 13 | 2000, mis en ligne le 16 juin 2011, consulté le 21 septembre 2020. URL : http://journals.openedition.org/kernos/1319; DOI : https://doi.org/10.4000/kernos.1319 
Paola Ceccarelli, La pirrica nell'antichità greco romana. Studi sulla danza armata, Pisa-Roma, Istituti editoriali e poligrafici internazionali, 1998. 1 vol. $29,5 \times 21 \mathrm{~cm}, 274$ p. , $24 \mathrm{pl}$. (Università degli studi di Urbino. Filologia e critica. Coll. diretta da Br. Gentili, 83). ISBN : 88-8147-140-X.

Les danses armées dans l'antiquité classique sont parmi les sujets les plus compliqués; elles font leur apparition déjà sur les vases de l'époque géométrique, mais le nom de «pyrrhique» n'est pas attesté avant le $v^{e}$ siècle av. J.-C. C'est précisément cette danse armée, sans doute la plus connue et la plus discutée, qui fournit le sujet du livre présenté ici. Il s'agit d'un long ouvrage de 232 p. qui vient s'ajouter à une bibliographie relativement riche concernant la pyrrhique antique, dans laquelle l'étude de J.C. Poursat, Les représentations de la danse armée dans la céramique attique, in $B C H, 92$ (1968), p. 550-615, joue un rôle fondamental.

partant d'une recherche sur la pyrrhique, l'auteur essaye de déterminer la signification des danses armées dans le cadre de la société et de la musique antiques. Elle se fonde sur des témoignages écrits et des documents iconographiques s'échelonnant depuis la période géométrique jusqu'à l'époque romaine.

L'étude contient neuf chapitres. Même si cela n'apparaît pas dans la table des matières, le livre est en fait divisé en deux parties : la première, qui comprend les chapitres II-V, traite de la répartition géographique des danses armées; la seconde, qui comprend les chapitres VI-IX, s'occupe des problèmes généraux de ces danses, concernant notamment leurs noms, la musique d'accompagnement et leurs sujets spécifiques.

Le premier chapitre est une introduction au sujet commençant par une définition générale de la danse, fondée sur une méthode anthropologique fonctionnaliste et accordant une importance particulière au contexte dans lequel la danse est placée. Cette section est complétée par des remarques sur la signification de la danse dans la culture grecque. Suit une présentation des danses armées et une analyse de leur fonction éducative d'après les sources anciennes. L'auteur se réfère ensuite à la relation entre les danses armées et la guerre ainsi qu'à la grande variété des traditions qui les concernent. Le chapitre se termine par une présentation des listes de danses armées chez Athénée et chez Pollux, et par une définition de la pyrrhique, terme qui se réfère, selon Athénée, à plusieurs formes de danse armée. L'auteur conclut que ce terme peut désigner à la fois une danse particulière et une catégorie assez large de danses armées. Il est souvent difficile de distinguer les deux sens.

Les chapitres II-V abordent le sujet de la danse armée sous un aspect géographique et examinent sa présence dans diverses régions. Le chapitre II concerne la pyrrhique en Attique : d'après les témoignages écrits et les documents iconographiques il semble qu'on la dansait aux Panathénées, mais qu'elle était aussi liée au thêâtre (Phrynique) et à des fêtes religieuses comme les Apatouries. L'analyse iconographique révèle l'existence d'une pyrrhique funéraire, de compétitions de danse, de performances dans les banquets et parmi les femmes, tandis que la présence occasionnelle de satyres et de ménades dans le contexte de la pyrrhique suggère une relation de cette danse avec le monde dionysiaque. Il semble que l'on dansait une pyrrique d'amazones en honneur d'Artémis. Divers schémas choréoraphiques relatifs à la pyrrhique sont examinés. Le recoupement des sources écrites et iconographiques mène la recherche de l'auteur sur la côte est de l'Attique, à Halai Araphénides, où se tenait la fête des Tauropolia pour Artémis. Le chapitre III examine la pratique de la pyrrhique dans d'autres régions de la Grèce propre, notemment en Eubée, en Mégaride, dans le Péloponnèse et en Crète. Le chapitre IV nous conduit vers l'est, en Asie Mineure (Xanthos, 
Aphrodisias) et dans les îles voisines de la côte (Cos, Rhodes), tandis que le chapitre $\mathrm{V}$ examine les danses armées dans la péninsule italienne, en Étrurie, en Lucanie, en Campanie et à Rome. Le chapitre VI contient une longue discussion sur le terme "pyrrhique » et sa signification.

Les chapitres suivants (VII-IX) forment la deuxième partie de l'étude. Dans le chapitre VII l'auteur examine les éléments relatifs à la musique et au rythme de la pyrrhique (qui est aussi une forme métrique) ainsi que les instruments musicaux qui accompagnaient cette danse. Le chapitre VIII est dédié au contenu des danses armées et à leurs relations avec différents sujets mythologiques. L'auteur s'efforce de déterminer la signification que peut assumer la danse dans le mythe. Le chapitre IX est en quelque sorte la suite du chapitre VIII, car il examine le rôle de la danse armée dans le théâtre et la pantomime. À la fin il y a un développement sur le costume des danseurs de la pyrrhique.

La recherche porte sur un matériel aussi étendu que varié, littéraire, épigraphique et iconographique et elle s'efforce de tenir compte de diverses approches méthodologiques afin d'éclairer le contenu et la signification des danses armées dans l'antiquité. C'est une entreprise complexe de grande envergure qui demande un inventaire systématique du matériel et une bonne connaissance de la bibliographie. Malgré la diligence dont elle témoigne, la présentation a le défaut d'être souvent répétitive et de proposer des interprétations parfois trop générales et schématiques des témoignages sans tenir compte des écarts chronologiques. L'auteur parle par exemple (p. 89) de la double identité de la pyrrhique " polverizzata fra centro e margini, fra maschile e feminile, fra guerra giovanile e combattimento oplitico... ". L'analyse de la danse armée en Attique en particulier contient d'intéressantes observations, mais elle présente dans certains cas des lacunes de connaissances qui entrainent l'auteur à formuler des opinions infondées : aux p. 61 et 63, elle maintient que le coffret (cofanetto) présent dans cer taines représentations montrant des femmes dansant la pyrrhique se réfère au mariage; elle méconnaît ainsi le fait que ce récipient, présent dans plusieurs scènes de gynécée où les femmes font de la musique et dansent, contient souvent des textes poétiques. Sa conclusion que la scène sur l'hydrie de Florence (p. 61) est relative aux noces de Pélée et de Thétis et aux rites de passage est également arbitraire (une interprétation différente est proposée par A. Goulaki Voutira, Observations on Domestic Music Making in Vase Paintings of the Fifth Century B.C., in Imago Musicae, VIII [1991], p. 73-90). Les rares omissions bibliographiques,

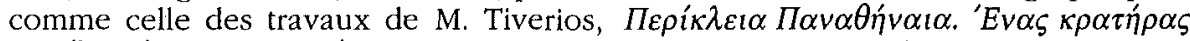

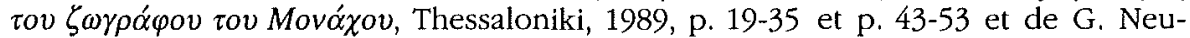
mann, in U. Hausmann (éd.), Der Tübinger Waffenläufer, Tübingen, 1977, p. 31 sq. sur les juges de concours (p. 50) ou les courses armées (p. 58), ne portent pas préjudice à l'utilité de ce travail riche et conciencieux qui pose des questions à plusieurs niveaux. Le lecteur risque cependant d'être déconcerté par un défaut mineur, celui de citer les images dans les notes et non dans le texte.

En conclusion il s'agit d'un instrument de travail utile dans le domaine de l'étude de la danse antique, où, malgré la riche bibliographie, les travaux systématiques sont encore peu nombreux. 